\title{
REVIEW
}

\section{Influence of type of management of transverse sacral fractures on neurological outcome. A case series and review of literature}

\author{
CU Dussa and BM Soni \\ Regional Spinal Injuries Centre, Southport District General Hospital, Southport, UK
}

\begin{abstract}
Study design: A retrospective study with review of literature.
Objectives: (1) To report the management and outcome of a series of six cases of transverse sacral fractures. (2) To review the literature and see the influence of the type of treatment on the neurological outcome, in particular, of the bladder and bowel and to identify the possible factors determining this outcome.

Setting: Regional spinal injury Unit, Southport, UK.

Methods: Six patients with transverse sacral fractures were identified and their case notes were reviewed. The follow-up period was 4 to 17 years. A review of literature was carried out and publications that reported the outcome of function of bowel and urinary bladder were considered for the study.

Results: Five of our six patients had cauda equina syndrome. Five cases were managed conservatively and one case surgically. Bladder and bowel function did not improve in three out of five cases. A review of English literature identified 18 (60 cases) of the 25 publications mentioning the outcome of bladder and bowel function. Of these, 47 cases were managed surgically, with the improvement of bladder and bowel function in 34 cases. Rest of the 13 cases were treated conservatively, with the improvement of bladder and bowel function in 10 cases.

Conclusion: There is no statistical evidence of benefit of either surgical or conservative management on the outcome of bladder and bowel function. Presence of a severe angulation, displacement of fracture and neurotomesis dictate poor prognosis.

Spinal Cord (2008) 46, 590-594; doi:10.1038/sc.2008.59; published online 10 June 2008
\end{abstract}

Keywords: sacrum; transverse fracture; cauda equina syndrome

\section{Introduction}

Transverse sacral fractures are uncommon injuries. This fracture was reported by Purser in $1969 .^{1}$ They constitute about $0.1 \%$ of polytrauma patients. ${ }^{2,3}$ The position and strength of sacrum makes it less vulnerable to fracture. Although Purser did not mention a neurological deficit in his original description, subsequent description of these fractures by several authors had high incidence of neurological damage and often associated with poor prognosis. Subtle neurological deficits complicated by frequently associated poly-trauma make these fractures difficult to detect at the time of presentation. This reminds us of Kinnier Wilson quotes 'the cauda equina is too complex a structure for simplicity of syndrome...' . ${ }^{4}$ These are classified as transverse Zone III fractures by Dennis et al. ${ }^{5}$ The neurological involvement involves motor and sensory function as well as paralysis of bladder and bowel function. The motor and sensory paralysis being typically subtle, the bladder and bowel paralysis are distressing and have an impact on the

Correspondence: CU Dussa, Pippinger Strasse 187, Munich 81247, Germany. E-mail: dussacu1@web.de

Received 8 October 2007; revised 24 March 2008; accepted 16 April 2008; published online 10 June 2008 quality of life. We report six cases of transverse fractures of sacrum that presented to our centre and reviewed the relevant literature to see whether managing these fractures conservatively or operatively have an effect on the outcome of bladder and bowel function.

\section{Materials}

The cases were identified from our database at Regional spinal injuries centre. We reviewed the case notes for medical history, diagnosis, investigations, treatment and outcome. The details of the patients are presented in Table 1 .

We also reviewed the English literature using Pubmed search engine using search words, sacral fractures, transverse sacral fractures and cauda equina syndrome. This resulted in 25 publications. The inclusion criteria for selection of a publication into our study is the mention of (a) the outcome of bladder and bowel function and (b) the outcome of motor and sensory function. Those publications that did not make specific reference to the outcome of the bladder and bowel function or mentioned only the outcome of the motor and sensory function were excluded. 
Table 1 Patient details

\begin{tabular}{|c|c|c|c|c|c|c|}
\hline Case & Age/sex & Year & Mechanism & Fracture level & Other fractures & Diagnosis \\
\hline 1 & $34 / \mathrm{M}$ & 1988 & Assault & S3/S4 & None & X-ray \\
\hline 2 & $23 / \mathrm{M}$ & 1991 & Fall from height & $\mathrm{S} 2 / \mathrm{S} 3$ & D7 vertebrae & $\mathrm{CT}$ \\
\hline 3 & $42 / \mathrm{M}$ & 1995 & RTA & S2 & $\begin{array}{l}\text { 1. Transverse process } L 5 \\
\text { 2. Pubic ramus }\end{array}$ & MRI \\
\hline 4 & $45 / \mathrm{F}$ & 2000 & Fall from height & S2 & Pubic ramus & MRI \\
\hline 5 & $35 / \mathrm{M}$ & 2001 & Fall from height & S2 & None & X-ray \\
\hline 6 & $58 / F$ & 2002 & Fall from height & S2 & Pubic ramus & $\mathrm{CT}$ \\
\hline
\end{tabular}

Abbreviations: CT, computerised tomography; MRI, magnetic resonance and imaging; RTA, road traffic accident.

\section{Results}

Over a period of 17 years, we identified six cases of transverse sacral fractures at our regional spinal injuries centre. There were four males and two females in the study group. The aetiology of the injury was fall from height in four cases, road traffic accident and assault in one case each. The diagnosis was delayed in five out of six cases due to the presence of other conspicuous injuries. Fracture of the sacrum occurred at the level of S2 in five cases. In the sixth case, the fracture occurred at the level of S3/S4 level. In addition to the sacral fractures, other fractures were present in four of the six cases. These included pubic ramus fracture in three cases and stable wedge compression fracture (Denis A) of dorsal spine at the level of D7 in one case. The post-injury neurology has been summarised in Table 2 .

Of the six cases, five of them had cauda equina syndrome at the time of presentation. In one patient, there was no neurological deficit. While the bladder and bowel and motor paralysis was present in five cases, sensory involvement was present only in four cases. Classifying the neurological deficit according to the ASIA impairment scale led to the following: four-complete (A), one-incomplete (B) and one-normal (E). Five cases were treated conservatively with bed rest for 6 weeks. In addition, Warfarin, TED stockings and in-bed mobilization were instituted to prevent the occurrence of deep venous thrombosis. After 6 weeks of conservative management, the patients were gradually mobilized depending on pain. Only in one case a surgical decompression was performed.

The period of follow-up was from 4 years to 17 years. Complete recovery of the bladder and bowel occurred in one case, whereas in four cases the recovery was incomplete to poor. Motor power improved in all five cases. This improvement was reflected both in improvement of the Gait and strength of Gluteii muscles as well as ankle and knee flexors. Sensory recovery was complete in one case and incomplete in three cases with persistence of perineal hypoesthesia. Recovery of sexual function was incomplete in two of the three male patients. The one patient without neurological deficit did not deteriorate during the conservative treatment. A summary of the treatment and neurological outcome of these cases are given in Table 3 .

Pubmed search of English literature with the abovementioned key words yielded 25 publications. Apart from a few case series, a majority of these publications are case
Table 2 Post-injury neurological deficit

\begin{tabular}{lllll}
\hline Case & Sensory & Motor & Anal tone/reflex & Bladder \\
\hline 1 & Normal & Bilateral S1-S4 & Absent & Atonic \\
2 & Bilateral S2-S4 & Bilateral S1-S4 & Absent & Atonic \\
3 & Bilateral S1-S4 & Bilateral S1-S4 & Absent & Atonic \\
4 & Bilateral S2-S4 & Bilateral S1-S4 & Absent & Atonic \\
5 & Normal & Normal & Normal & Normal \\
6 & Bilateral S2-S4 & Bilateral S1-S4 & Absent & Atonic \\
\hline
\end{tabular}

Table 3 Neurological recovery

\begin{tabular}{llllll}
\hline Case & Management Sensory & Motor & Bowel & Bladder & Sexual \\
\hline 1 & Conservative Normal & Normal & Good & Poor & Incomplete \\
2 & Conservative Incomplete Normal & Poor & Poor & Good \\
3 & Operative & Incomplete Normal & Poor & Poor & Incomplete \\
4 & Conservative Normal & Grade 3/5 & Poor & Good & - \\
5 & Conservative Normal & Normal & Good & Good & Good \\
6 & Conservative Incomplete & Grade 3/5 & Good & Fair & - \\
\hline
\end{tabular}

${ }^{\mathrm{a} G o o d}$, normal bladder control; Fair, urge incontinence; Poor, self-catheterization.

${ }^{\mathrm{b}}$ Good, normal habit; Poor, manual evacuation.

reports. Seven publications were excluded, as they did not fit into the inclusion criteria, leaving 18 publications, which were included into the study (Table 4 ). All the 18 publications were searched for the mode of injury, post-injury neurological status, intervention and post-treatment neurological status.

The resulting data were tabulated in a $2 \times 2$ contingency table. As frequency of one of the variable in our data is less than 5 , the data were analysed using the Yates $\chi^{2}$ test. $P$-value of less than 0.5 was considered significant. Thereafter $95 \%$ confidence interval and odds ratio was calculated for a favourable outcome between operative and conservative treatments using $2 \times 2$ contingency table. The Yates $\chi^{2}$ test indicated that there was no significant association $(P$-value $=0.981)$ between the method of treatment and the bladder and bowel outcome. Furthermore, the odds ratio of surgery improving the bladder and bowel function is 0.78 with a 95\% confidence interval of $0.18-3.31$. This analysis would support that operative intervention in comparison to the conservative treatment in treating these fractures, does not increase the chances of recovery of bladder and bowel function. 
Table 4 Reviewed publications

\begin{tabular}{|c|c|c|c|c|c|c|}
\hline \multirow[t]{2}{*}{ S. no. } & \multirow[t]{2}{*}{ Authors } & \multirow[t]{2}{*}{ Cases included } & \multicolumn{2}{|c|}{ Conservative } & \multicolumn{2}{|c|}{ Operative } \\
\hline & & & $B \& B$ improved & $B \& B$ not improved & $B \& B$ improved & $B \& B$ not improved \\
\hline 1 & Fountain & $6 / 6$ & 1 & & 5 & \\
\hline 2 & Roy-Camille & $9 / 13$ & & 1 & 1 & 7 \\
\hline 3 & Carl $^{6}$ & $1 / 1$ & & & 1 & \\
\hline 4 & Sapkas $^{7}$ & $1 / 2$ & & & 1 & \\
\hline 5 & Singh & $1 / 1$ & & & 1 & \\
\hline 6 & Byrnes & $2 / 2$ & 1 & & & 1 \\
\hline 7 & Kim & $6 / 7$ & & 1 & 4 & 1 \\
\hline 8 & Reynier & $2 / 2$ & & & 2 & \\
\hline 9 & Phelan & $4 / 4$ & 4 & & & \\
\hline 10 & Fardon & $1 / 1$ & & & 1 & \\
\hline 11 & Weaver & $1 / 1$ & & & 1 & \\
\hline 12 & Gibbons & $4 / 4$ & 1 & & 2 & 1 \\
\hline 13 & Charutini $^{8}$ & $1 / 1$ & 1 & & & \\
\hline 14 & Savolaine & $1 / 1$ & & & 1 & \\
\hline 15 & Denis & $11 / 13$ & 2 & 1 & 7 & 1 \\
\hline 16 & Taguchi & $4 / 5$ & & & 4 & \\
\hline 17 & Ebraheim & $4 / 4$ & & & 2 & 2 \\
\hline \multirow[t]{2}{*}{18} & Yasuda $^{9}$ & $1 / 1$ & & & 1 & \\
\hline & Total & $60 / 69$ & 10 & 3 & 34 & 13 \\
\hline
\end{tabular}

Abbreviation: B\&B, bladder and bowel.

Note: the discrepancy between the cases and the sum of the cases in the outcome is due to the fact that the authors did not mention the outcome in some of their cases.

\section{Discussion}

Transverse sacral fractures are uncommon. In published series these fractures constituted about $2-4 \%$ of pelvic fractures, $0.2 \%$ of spinal fractures and $15 \%$ of fractures of sacrum. ${ }^{5,10-12}$ In the absence of osteoporosis these fractures are caused by high impact trauma. Review of the published cases shows that about $62 \%$ of these injuries are caused by fall from height and the rest as result of automobile accident. Similar observations are made by Wakeley et al. ${ }^{13}$ RoyCamille noted in his series that $84 \%$ of these fractures caused by fall from height and called them as 'jumpers fracture'. ${ }^{14}$ Owing to this high impact, these fractures are often associated with multiple fractures sometimes involving spine, pelvis or both. ${ }^{5,14}$ It has been pointed out that about $25 \%$ of those patients who sustain this injury as a result of fall have a thoraco-lumbar burst fracture. ${ }^{13}$ Reviewing the published literature, we found a $21 \%$ incidence of associated pelvic fractures and $38 \%$ (18 cases) association of spinal fracture at a higher level. These proximal spinal fractures occurred at the level of dorsal, dorsolumbar or lumbar spine. A spinal cord injury (paraplegia) at the level of proximal spinal fracture was found in $61 \%$ (11 cases) of the fractures. Rest of the proximal spinal injuries were not associated with paraplegia or neurological injury. The absence of gross neurological signs, attention drawn by other fractures, presence of a proximal spinal fracture and a resulting paraplegia and lack of suspicion, make these fractures susceptible for late diagnosis. ${ }^{15-17}$ Such late diagnosis of these fractures has been observed by several authors. Although Schmidek et al. and Rowell ${ }^{18,19}$ noted the fracture commonly at the level of S3, we noted from the available literature that the fracture commonly occurred at the S1-2 level. We also noted that the fractures are more common in the proximal sacral segment (that is, between S1 and S3) than the distal sacral segment (that is, between S3 and S5).

Despite our hospital being a regional referral centre for spinal injuries, we have so far treated only six cases in last 17 years. This speaks of the rarity of the injury. While fall from height was responsible for this injury in four of six cases, direct injury was the cause in two cases. The level of fracture was in the proximal sacral segment in five of the six cases. We classified these fractures according to the mechanism of injury and found three-flexion, two-extension and one-undisplaced fractures. ${ }^{14}$ All these patients were referred to us from other centres. In consistence with several citations in the literature, we noted a delay in the diagnosis of the fracture at the presenting hospital in five out of six cases.

Conventional pelvic X-rays taken as a part of poly-trauma screen are not enough to diagnose these fractures. ${ }^{14}$ This is due to several factors such as superimposition of the bowel loops on sacrum, superimposition of the bones especially the upper sacrum, osteoporosis (in elderly). The fracture is best viewed on the lateral view of sacrum. However, it is frequently difficult to obtain such a view due to pain and associated injuries in Accident and Emergency department. ${ }^{20}$ Fracture of transverse process of L5 vertebra on a AP view may give clue to a fracture of upper sacrum. ${ }^{1}$ Myelography although described is invasive and outdated. ${ }^{5,14,15,17}$ Noninvasive investigations such as CT (computerised tomography) and MRI (magnetic resonance and imaging) scan increases the detection rate. ${ }^{20,21}$ MRI has an added advantage of showing the type of neural injury in addition to the fracture. $^{20}$

Neurological injury is a serious consequence of transverse sacral fracture, which was found in five out of six $(83 \%)$ of patients in our series. Literature, however, quotes a lower 
incidence ranging between 56.7 and 63.6\%.5,10,22 Presence of avulsion of L5 transverse process, comminuted sacral fracture, and bilateral vertical sacral fractures increase the risk of neurological injury and therefore should prompt a keen examination for a subtle neurological deficit. ${ }^{22}$ Bladder is commonly involved. This is followed by involvement of bowel and sexual function. 5,10,11,14-17,21,23,24 The segmental innervation of the above organs being from S2,3,4 may be injured by fractures at or below S1 level. Such neurological injury is either a neuropraxia or neurotomesis depending upon displacement and angulation of the fracture. The motor paralysis involves the pelvic floor muscles $(\mathrm{S} 2,3,4)$, the hip extensors (L5, S1,2), Hamstring muscles (L5, S1,2), flexors of ankle and toes $(\mathrm{S} 1,2)$ and intrinsic muscles of foot $(\mathrm{S} 2,3)$. The sensory impairment is in the perineal region, back of leg and thigh, therefore escapes an improper examination. The bladder involvement in a complete cauda equina injury is typically hypotonic low-pressure lower motor neuron bladder confirmed by video urodynamics. The presence of an upper motor neuron bladder is indicative of a higher spinal lesion.

The options available for treating sacral transverse fractures are both surgical and conservative. ${ }^{11,15,16,21}$ The conservative treatments include traction, ${ }^{1}$ plasters ${ }^{25}$ and bed rest. ${ }^{26}$ Although traction and plasters are mentioned in the literature, these options are only of historical value. Irrespective of the method of conservative intervention used, they need prolonged bed rest. Operative intervention is based on early decompression of the nerves and stabilization of the fracture. Delayed decompression may also be useful. ${ }^{5}$ This facilitates pain-free early mobilization of the patient. ${ }^{5,11,23,24,26}$ Even though pain relief and early ambulation are benefits of operative fixation, ${ }^{10}$ its role in neurological improvement of bladder and bowel is yet to be established. Improvement in motor function was found in most series treated by both conservative and operative methods. Reviewing all the publications chosen for this study, the sensory-motor improvement was found in $71 \%$ of the conservatively treated and $84 \%$ of the surgically treated cases. Improvement of bladder and bowel function was found in about $77 \%$ of the conservatively treated and $72 \%$ of the surgically treated cases. While the motor power improved in all our patients, the bladder and bowel function improved only in $40 \%$ of the cases. This is quite lower compared to the outcome mentioned above.

We asked ourselves the question, are there any predictive factors for the recovery/improvement of neurological function? We reviewed our own six cases for type of sacral fracture, level of injury, higher level of injury, ${ }^{14}$ state of sacral nerves. ${ }^{2,5,12,27}$ We found three flexion fractures producing severe internal angulation deformity possibly severely compromising the nerves, and two extension type of fractures, where there was complete dorsal displacement of the distal fracture possibly resulting in tear of nerves. Such neurotomesis could be documented in one case (case 3) that was operated. In one case, the MRI showed possible partial tear of sacral nerves on one side (case 4 ). In the rest of the cases there was no documentation of state of nerves. We looked for the same criteria to determine if they are influencing factors that determine the outcome. These criteria unfortunately could not be uniformly applied to all the publications, as several of them did not include the type of sacral fracture. Therefore, no association could also be drawn between the type of sacral fracture and the neurological outcome. Similarly, no association could be made between impact of a dorsolumbar fracture on the outcome of sacral fracture due to small number of patients. In the reviewed literature only six cases (clearly mentioned) with dorso-/lumbar fractures and paraplegia that were operated along with sacral fracture, of which complete neurological recovery was found in four cases. Of the 18 publications chosen for the review, 14 mentioned the state of nerves during surgical decompression. Of these, 30 cases had neuropraxia and 13 cases had either complete or partial neurotomesis. In two cases there was transaction of spinal cord at the level of dorsolumbar spine. With the exception of five cases, most of the operated cases, which showed neuropraxia improved in bladder and bowel function. In cases of neurotomesis, although some authors made an attempt to repair the nerves, no neurological recovery was found. We found in our case series as well as in others a displaced and severely angulated sacral fractures dictated poor prognosis. ${ }^{14,23,27}$ In such cases, an operative intervention of the transverse sacral fracture may not bring much recovery and perhaps may only facilitate early mobilization of the patient. According to the available literature, it cannot be clearly stated if the presence of a higher level dorsal, dorsolumbar or lumbar fracture with paraplegia influences the outcome of the sacral fractures. It only seems to be logical to decompress the proximal vertebral injury before decompressing the sacrum. However, in minimally displaced fractures, where there may be a neuropraxia, surgical decompression, although there is no statistical evidence, may be of value to facilitate early recovery of nerves. In how many cases in our series an operative intervention could have helped in neurological improvement is subject to discussion.

We need more number of cases with clear description of the type of sacral fracture, associated injuries and good documentation of the neurological injury and outcome to draw guidelines for management of these fractures in future. Currently, we can only suggest that observation for continuity of nerves in the cauda equina on MRI should be looked for before planning for surgery.

\section{Conclusions}

A transverse sacral fracture has a high incidence of neurological deficit. Diagnosis is often delayed due to insufficient neurological examination and lack of awareness of this possible injury. Although majority of the fractures in the literature are managed surgically, management remains debatable. While following this injury, the outcome of bladder and bowel paralysis was found to be poor, currently there is no statistical evidence of an advantage of a operative treatment over a conservative treatment in terms of improvement of bladder and bowel function. As few studies which documented the neurotomesis did not have any 
recovery of the function, it may be advisable to base the management of these fractures based on the MRI findings for the continuity of the nerves. Furthermore, due attention should be paid to the anatomy of the spinal cord when a fracture is present at a higher level. Such fractures can influence the final outcome of the treatment of sacral fractures.

\section{Acknowledgements}

We would like to acknowledge Dr Morris K, senior lecturer and researcher, school of biomedical sciences, Cardiff University and Dr Rajesh EK for their input in the statistical analysis of the data.

\section{References}

1 Purser DW. Displaced fracture of the sacrum. Report of a case. J Bone Joint Surg (Br) 1969; 51: 346-347.

2 Byrnes DP, Russo GL, Ducker TB, Cowley RA. Sacrum fractures and neurological damage. Report of two cases. J Neurosurg 1977; 47: 459-462.

3 Favuzzi E. Le fratture isolate del sacro. G Med Milit 1956; 106: 739-744.

4 Bonin JG. Sacral fractures and injuries to the cauda equina. J Bone Joint Surg 1945; 27: 113-127.

5 Denis F, Davis S, Comfort T. Sacral fractures: an important problem. Retrospective analysis of 236 cases. Clin Orthop 1988; 227: 67-81.

6 Carl A, Delman A, Engler G. Displaced transverse sacral fractures. A case report, review of the literature, and the CT scan as an aid in management. Clin Orthop Relat Res 1985; 194: 195-198.

7 Sapkas G, Makris A, Korres D, Kyratzoulis J, Meleteas E, Antoniadis A. Anteriorly displaced transverse fractures of the sacrum in adolescents: report of two cases. Eur Spine J 1997; 6: 342-346.

8 Chiaruttini M. Transverse sacral fracture with transient neurologic complication. Ann Emerg Med 1987; 16: 111-113.

9 Yasuda T, Shikata J, Iida H, Yamamuro T. Upper sacral transverse fracture. A case report. Spine 1990; 15: 589-591.

10 Gibbons KJ, Soloniuk DS, Razack N. Neurological injury and patterns of sacral fractures. J Neurosurg 1990; 72: 889-893.
11 Fountain SS, Hamilton RD, Jameson RM. Transverse fractures of the sacrum. A report of six cases. J Bone Joint Surg (Am) 1977; 59: 486-489.

12 Reynier Y, Grisoli F, Delarque A, Vigouroux RP. Fracture dislocation of the sacral spine with cauda equina lesion. Report of two cases. Neurochirurgie 1982; 28: 25-32.

13 Wakeley CPG. Fractures of the pelvis: an analysis of 100 cases. Br J Surg 1929; 17: 22-29.

14 Roy-Camille R, Saillant G, Gagna G, Mazel C. Transverse fracture of the upper sacrum. Suicidal jumper's fracture. Spine 1985; 10: 838-845.

15 Phelan ST, Jones DA, Bishay M. Conservative management of transverse fractures of the sacrum with neurological features. A report of four cases. J Bone Joint Surg (Br) 1991; 73: 969-971.

16 Sabiston CP, Wing PC. Sacral fractures: classification and neurologic implications. J Trauma 1986; 26: 1113-1115.

17 Weaver Jr EN, England GD, Richardson DE. Sacral fracture: case presentation and review. Neurosurgery 1981; 9: 725-728.

18 Schmidek HH, Smith DA, Kristiansen TK. Sacral fractures. Neurosurgery 1984; 15: 735-746.

19 Rowell CE. Fracture of sacrum with hemisaddle anaesthesia and cerebro-spinal fulid leak. Med J Aust 1965; 14: 16-19.

20 Savolaine ER, Ebraheim NA, Rusin JJ, Jackson WT. Limitations of radiography and computed tomography the diagnosis of transverse sacral fracture from a high fall-A case report. Clin Orthop Relat Res 1991; 272: 122-126.

21 Ebraheim NA, Biyani A, Salpietro B. Zone III fractures of the sacrum - A case report. Spine 1996; 21: 2390-2396.

22 Pohlemann T, Gansslen A, Tscherne $H$. The problem of the sacrum fracture. Clinical analysis of 377 cases. Orthopade 1992; 21: $400-412$.

23 Singh H, Rao VS, Mangla R, Laheri VJ. Traumatic transverse fracture of sacrum with cauda equina injury - a case report and review of literature. J Postgrad Med 1998; 44: 14-15.

24 Taguchi T, Kawai S, Kaneko K, Yugue D. Operative management of displaced fractures of the sacrum. J Orthop Sci 1999; 4: 347-352.

25 Rotini R, Miceli D, Noia F, Fontanesi G. Transverse fracture of the second sacral vertebra: description of a clinical case. Chir Organi Mov 1998; 83: 309-314.

26 Fardon DF. Displaced transverse fracture of the sacrum with nerve root injury: report of a case with successful operative management. J Trauma 1979; 19: 119-122.

27 Kim MY, Reidy DP, Nolan PC, Finkelstein JA. Transverse sacral fractures: case series and literature review. Can J surg 2001; 44: 359-363. 